the interposition of a blackened brass disc slightly larger than the solar disc.

The resulting negatives showed distinct halos around the disc, and, notwithstanding the fact that some time elapsed between the successive exposures, these halos exhibited the same form, thus testifying to their solar origin. Some of the negatives were photographically intensified by repeated copying, and reproductions of them were submitted to the academy. In presenting the communication M. Janssen-to whom M. Hansky acknowledges his obligations for assistance and advice-stated that "the photographs actually show the solar corona with an intensity and a perfection only known on the photographs obtained during total eclipses" (Comptes rendus, No. 12).

Search-ephemeris for Tempel's First Periodic Comet (1867 II).-Although Tempel's first comet has not been seen during its last three perihelion passages, i.e. since I879, M. A. Gautier, of the Geneva Observatory, thinks that the probability of its re-discovery this year is great enough to justify a careful search. For this reason he re-publishes, in No. 4008 of the Astronomische Nachrichten, the elements he prepared for the 1898 apparition, reduced to the mean equinox of 1905.0 . As the probable time of perihelion is somewhat uncertain, he gives three ephemerides, extending from March $3 \mathrm{I}$ to July 13 , in which this time is reckoned as May 2.5, April 20.5, and April 8.5 respectively, the mean date being the most probable. The declination varies from $-16^{\circ}$ to $-31^{\circ}$, so that the more southerly observatories are more likely to be successful in the research.

Right Ascensions of 2 I20 Southern Stars.-In an appendix to "Observations made at the Hong Kong Observatory during 1903 ," Prof. W. Doberck, the director, publishes the right ascensions of 2120 southern stars for the epoch 1900 , as determined from observations made by Mr. J. I. Plummer and himself during the years 1898 to I904.

The observations were made with a 3 -inch Simms semiportable transit instrument, which, together with the method of reduction and the comparisons with other catalogues, is briefly discussed in the director's preface.

In the catalogue itself, the number of the star as given in Lacaille, or Stone, or both, the R.A., epoch and magnitude, the variation of the R.A. from Stone's corresponding value, the proper motion, and several other particulars are given for each star.

The Iris Diaphragm in Astronomy.--In a communication to the French Academy of Sciences, M. Salet states that he has recently and usefully adapted the iris diaphragm to a telescope in which the magnification employed is 500. The diaphragm is placed very near to the plane of the micrometer wires in front of the field lens, and its raison d'être is to prevent the light from the sky, and from the illumination of the wires, from reaching the eye when feeble objects are being observed, the diaphragm being closed by an external cylinder when the object has been brought to the centre of the field. By reducing the extent of the micrometer wires, the diaphragm also reduces, or eliminates, the effect of astigmatism when observations of double stars are being made (Comptes rendus, No. 9).

Constancy of "Spark" WaVe-Lengths.-A question which is of first importance to those observers engaged in stellar line-of-sight work, viz. that of the constancy of wave-lengths in spark spectra taken under various conditions of discharge, has recently been re-investigated by Mr. G. W. Middlekauff at the Johns Hopkins University. A detailed description of the apparatus and methods employed, together with the results obtained, appear in No. 2, vol. xxi., of the Astrophysical Journal.

Mr. Middlekauff used a concave Rowland grating of 20,000 lines to the inch and a focal length of $2 \times \cdot 5$ feet. The self-induction in the spark circuit could be varied from 0.00007 to 0.0012 of a henry, and the capacity from 0.0085 to 0.0739 of a microfarad, and the results obtained afford strong evidence that in the case of a spark discharge in air, at atmospheric pressure, no "shift" in wave-length is produced by variations of self-induction or capacity NO. I 849 , VOL. 7 I] within the above limits. A further result obtained was that the analogous wave-lengths in the arc and the spark spectra of the same elements are not measurably different.

\section{STATISTICS OF VARIATION.}

A PAPER consisting mainly of a large number of elaborate records bearing on the important subject of variation has recently been issued by the Washington Academy of Sciences. The data, which have been collected with much care and industry, cannot fail to be of high interest to all students of evolution. They afford an excellent example of the peculiar value of insect studies in reference to many difficult problems in biology-a point which has lately received fresh emphasis from Prof. Poulton's valedictory address as President of the Entomological Society of London.

The authers start with an "Introduction," in which they declare thcir " belief in the marked betterment and effectiveness of practically all variation study when pursued from the point of view of the biometrician "; adding, however, that "from the writers' point of view the study of variation is a phase of biology, and not of mathematics." Dealing with the special advantages presented by insect data in this inquiry, they assert that the phenomena of complete metamorphosis afford a luady means of distinguishing "variations which are strictly blastogenic from others which may be in large part acquired." This, it may be remarked, is only true under certain limitations. It is not the case, for instance, as the authors appear to think, that the imaginal colour-patterns of lepidoptera are uninfluenced by the conditions obtaining during individual development.

Coming now to the main substance of the paper, we find a series of short articles or sections giving statistics of variation in some two dozen species of insects. Among the structures thus dealt with are the venation and costal wing hooks in bees and ants, the venation in gnats, the colour-patterns of sundry beetles, wasps and bugs, the eye-spots of certain butterflies, the tibial spines, tarsal and antennal segments, tactile hairs and elytral striæ of other insects of various orders. In the case of the hive bee it is incidentally shown that the parthenogenetically produced drones are as subject to variation in their wings as are the workers of biparental ancestry. The results are in many cases graphically summarised in the form of the frequency polygon; and the "mode," " standard deviation," "index of variability," and "coefficient of variation" are duly reckoned and recorded in accordance with approved biometrical methods. It is interesting but not surprising to observe that the frequency curve is usually in fair correspondence with the law of error.

The paper ends with a section devoted to "general results." Here we think that too much is made of the difficulty of distinguishing between congenital variation and acquired modification. For practical purposes the distinction is usually obvious enough. A little later the authors observe, "The most satisfactory answer to the question of the hereditary transmission of acquired characters will come as the result of a quantitative (statistical) study of variations known to be blastogenic compared with a similar study of variations known to be acquired, both studies to be made on complete series of individuals bred under quantitatively determined life conditions." This seems to us somewhat like using a steam-hammer to crack an egg. It is not astonishing to find that there is little or no evidence of differing selection-value in the variable number of spots on the elytra of a ladybird; but it hardly seems clear that the authors are justified in claiming this fact, together with an apparent change of "mode" between the years I895 and x90r, as evidence in favour of " determinate variation." Before any such inference can properly be drawn, the question of possible correlation ought at least to be considered. The authors, however, arrive on the whole subject at the satisfactory conclusion that natural selection " is after all a logical necessity and undoubtedly an actual actively-regulative factor" in the formation of species.

F. A. D.

I "Studies of Variation in Insects." By Vernon L. Kellogg and Ruby G. Bell, of Leland Stanford Junior University. From the Proceedings of
the Washington Academy of Sciences, vol. vi. (Washington, D.C., x904) 